\title{
A GntR family transcription factor positively regulates mycobacterial isoniazid resistance by controlling the expression of a putative permease
}

\author{
Jialing $\mathrm{Hu}^{1 \dagger}$, Lei Zhao ${ }^{1+}$ and Min Yang ${ }^{2^{*}}$
}

\begin{abstract}
Background: Bacteria use transcriptional regulation to respond to environmental stresses. Specifically, exposure to antibacterial drugs is deemed to be an atypical stress, and altering transcriptional regulation in response to such stress can increase bacterial drug resistance. However, only a few transcription factors that regulate drug resistance have been reported.

Results: In the present study, a GntR family transcription factor, encoded by the MSMEG_0535 (Ms0535) gene, was shown to be an isoniazid (INH) resistance regulator in Mycobacterium smegmatis. When the Ms0535 gene was overexpressed, cells showed a significant increase in INH resistance. First, the interaction between Ms0535 and its own promoter was determined, and a conserved 26-bp palindromic DNA binding motif was identified using electrophoretic mobility shift and DNasel footprinting assays. Second, quantitative reverse transcription-PCR assays showed that Ms0535 acted as a transcriptional activator, and positively regulated its own expression, as well as that of a permease encoded by the MSMEG_0534 (Ms0534) gene. Similar to the case for the Ms0535 gene, a recombinant Ms0534-overexpressing strain also exhibited increased INH resistance compared with the wild-type strain. Furthermore, we showed that Ms0535 and Ms0534 deletion strains were more sensitive to INH than the wild-type strain. Interestingly, overexpressing Ms0534 in the Ms0535 deletion strain enhanced its INH resistance. In contrast, the Ms0534 deletion strain was still sensitive to INH even when Ms0535 was overexpressed. These findings suggest that Ms0534 is an effector protein that affects INH resistance in M. smegmatis.
\end{abstract}

Conclusions: In summary, the GntR transcriptional regulator Ms0535 positively regulates INH resistance by transcriptionally regulating the expression of the Ms0534 permease in M. smegmatis. These results improve our understanding of the role of transcriptional regulation in INH drug resistance in mycobacteria.

Keywords: Mycobacteria, GntR, Isoniazid, Permease

\section{Background}

Transcriptional regulation plays an important role in the bacterial response to environmental stresses. Antibacterial drugs are deemed to be atypical stressors, and changes in transcriptional regulation can increase bacterial drug resistance [1]. When exposed to such drugs, bacteria can undergo complex responses, such as inactivating the targets, modifying the drugs, or expressing drug efflux system proteins. Today, more than 20 transporter proteins

\footnotetext{
* Correspondence: yangmin@mail.hzau.edu.cn

${ }^{\dagger}$ Equal contributors

${ }^{2}$ College of Life Science and Technology, Huazhong Agricultural University, Wuhan 430070, China

Full list of author information is available at the end of the article
}

have been found to be associated with tetracycline efflux systems [2]. Bacterial drug efflux pumps can efflux drugs out of the cell, thereby lowering the drug concentration, and up-regulating the expression of membrane drug efflux pump proteins is the key mechanism by which bacteria increase their drug resistance [3]. Five different categories of drug transporters have been investigated: the ATP-binding cassette $(A B C)$ superfamily, the major facilitator superfamily (MFS), the multidrug and toxic compound extrusion (MATE) family, the small multidrug resistance (SMR) family, and the resistance/nodulation/cell division (RND) family [2].

MFS members are widespread, and they play important roles in drug transport in organisms ranging from bacteria 
to humans [4]. The MFS was first found to transport glucide, and, subsequently, drugs, phosphates, and oligosaccharides [5]. An MFS permease is directly involved in the bacterial drug efflux process [4]. Some bacterial transcription regulatory proteins, such as Bacillus subtilis BmrR, Staphylococcus aureus QacR, and Mycobacterium smegmatis LfrA, can regulate the expression of drug transporters by acting either as transcriptional repressors or activators of drug efflux genes [6-8]. However, our knowledge of the transcriptional factors that regulate anti-mycobacterial drug transporter-related genes is still limited.

Mycobacterium smegmatis is a relatively fast-growing and non-pathogenic mycobacterium, and it is frequently used as a model organism to study the gene regulatory mechanisms of M. tuberculosis $[9,10]$. Notably, the genome of M. tuberculosis encodes at least 20 possible drug efflux transporters [11]. Some of these transporter proteins play a role in mycobacterial resistance to isoniazid $(\mathrm{INH})$, rifampicin (RIF), tetracycline, and other antibiotics [12-14]. In comparison with M. tuberculosis, M. smegmatis has more drug efflux systems and transcription factors; however, the mechanisms by which they are regulated are largely unknown.

GntR family transcription factors, which are named after a gluconate operon repressor in B. subtilis, are widely distributed in bacteria $[15,16]$. GntR members contain a conserved N-terminal helix-turn-helix (HTH) domain for DNA-binding and diverse C-terminal domains [15]. The variable $\mathrm{C}$-terminal domain provides the basis for their classification into six subfamilies. Except for Ms2173, a global $\mathrm{Cu}^{2+}$-responsive transcription factor [17], members of the GntR/FadR subfamily have been rarely reported in $M$. smegmatis. GntR family members usually function as transcriptional repressors, although some of them are transcriptional activators. For instance, Serratia marcescens PigT [18], Enterococcus faecalis CitO [19], FadR [20], and McbR/YncC [21] from Escherichia coli, NorG from Staphylococcus aureus [22, 23], HpxS from Klebsiella pneumoniae [24], and B. subtilis GabR [25] were shown to act as transcriptional activators or repressor/activators.

In this study, we have shown that a GntR family transcription factor, encoded by the Ms 0535 gene, plays a role in M. smegmatis drug resistance. Ms0535 specifically bound its own promoter by recognizing a 26-bp palindromic sequence motif that is separated by four nucleotides. It regulates $M$. smegmatis INH resistance by functioning as a transcriptional activator that regulates the expression of the MFS permease gene Ms0534, which is located in the same operon. This is the first study to clarify the regulatory mechanism of the transcriptional factor Ms0535 and its role in M. smegmatis INH resistance.

\section{Results}

Mycobacterium smegmatis Ms0535 potentially contributes to mycobacterial INH resistance

To identify potential transcription factors that regulate drug resistance in $M$. smegmatis, we screened a transcriptional regulator library by spotting these recombinant strains onto plates containing INH $(20 \mu \mathrm{g} / \mathrm{mL})$. About 500 predicted regulatory genes in the genome of the $M$. smegmatis strain $\mathrm{mc}^{2} 155$ (Genbank accession number CP000480) were cloned into pMV261, and the recombinant plasmids were transformed into M. smegmatis. Transformant strains were spotted onto $7 \mathrm{H} 10$ medium containing $20 \mu \mathrm{g} / \mathrm{mL}$ INH. The recombinant plasmids were isolated from the INH-resistant $M$. smegmatis transformants, which enabled the positive regulator genes to be characterized by sequencing.

A hypothetical transcriptional factor, encoded by the Ms0535 gene, was isolated as a potential contributor to INH resistance in M. smegmatis. As shown in Fig. 1a, the mycobacterial strain transformed with pMV261Ms0535, which overexpresses Ms0535, was more resistant to $20 \mu \mathrm{g} / \mathrm{mL}$ INH than the wild-type strain transformed with the empty pMV261 vector (Fig. 1a). The evidence suggested that Ms0535 is potentially involved in regulating INH drug resistance in $M$. smegmatis. A sequence analysis revealed that Ms0535 contains an Nterminal winged HTH DNA-binding domain and a typical all-helical C-terminal domain belonging to the FadR subfamily [26]. The absence of the first $\alpha$-helix indicates that Ms0535 encodes a VanR-like regulator (Fig. 1b).

\section{Ms0535 binds its own promoter}

Several assays were performed to detect the DNA binding activity of Ms0535. First, a bacterial one-hybrid system [27] was used to detected protein-DNA interactions. The promoter region (500 bp upstream) of Ms0535, Ms0535p, and the unrelated promoter $M s 0540 \mathrm{p}$ (500 bp upstream of Ms0540) were cloned into the reporter vector $\mathrm{pBXcmT}$ [27] and co-transformed into reporter strains with pTRGMs0535. Co-transformants of both the positive control pTRG-Rv3133c/pBX-Rv2031p, as well as PTRG-Ms0535/ pBX-Ms0535p, grew very well in the screening medium (Fig. 2a). In contrast, no growth was observed for the selfactivated controls and the negative control (Fig. 2a). Thus, Ms0535 can bind to its promoter, Ms0535p .

Second, electrophoretic mobility shift assays (EMSAs) were conducted to show that Ms0535 binds to Ms0535p in vitro. As shown in Fig. $2 \mathrm{~b}, 0.03 \mathrm{nM}$ of the $M s 0535 p$ DNA substrate was co-incubated with different amounts (1-4 $\mu \mathrm{M})$ of his-tagged Ms0535 protein, and clear band shifts were observed, indicating the formation of DNAprotein complexes (Fig. 2b, lanes 1-4). In contrast, Ms0535 did not bind to the Ms0540p negative control promoter (Fig. 2b, lanes 5-8). Additionally, a competition assay was 


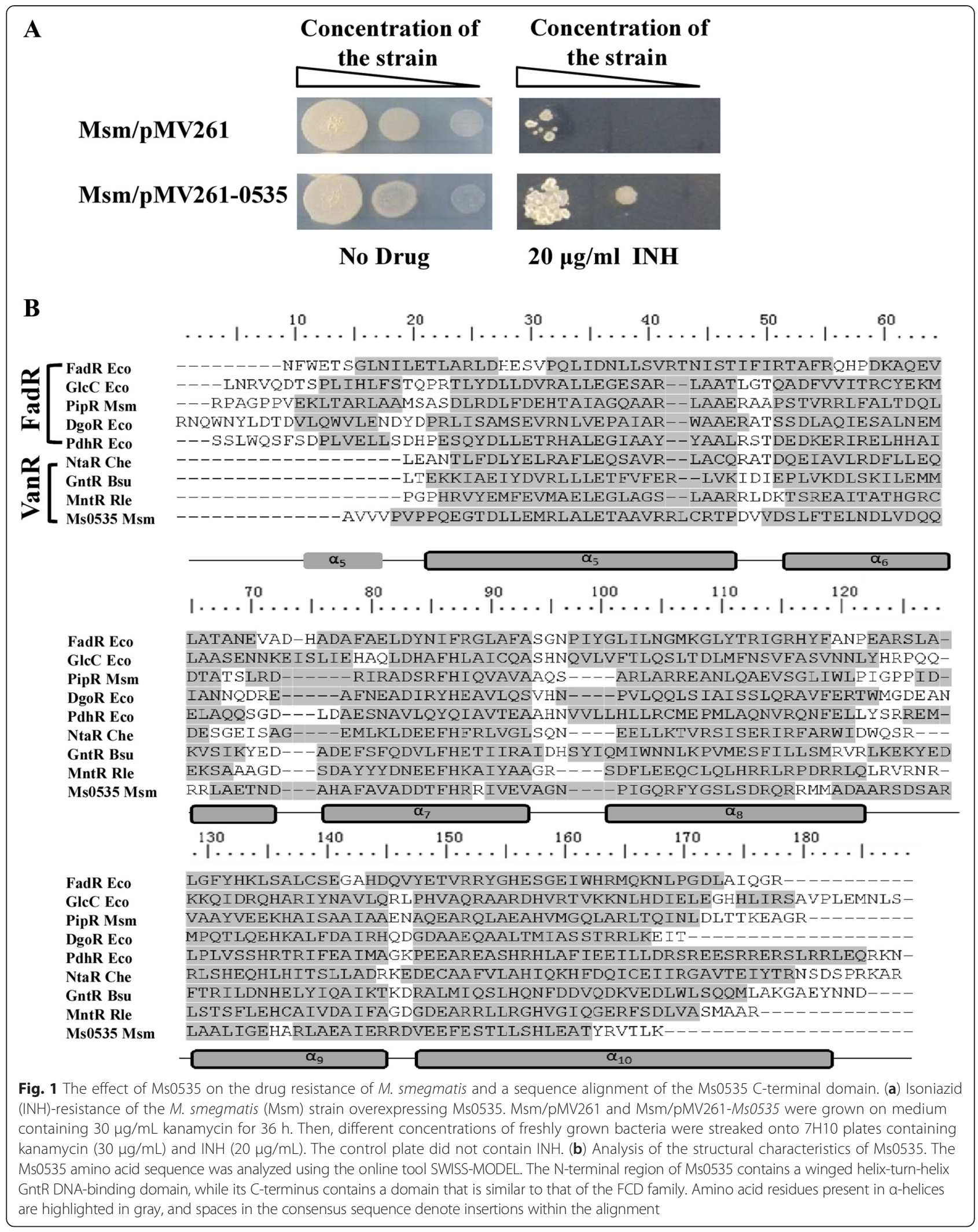




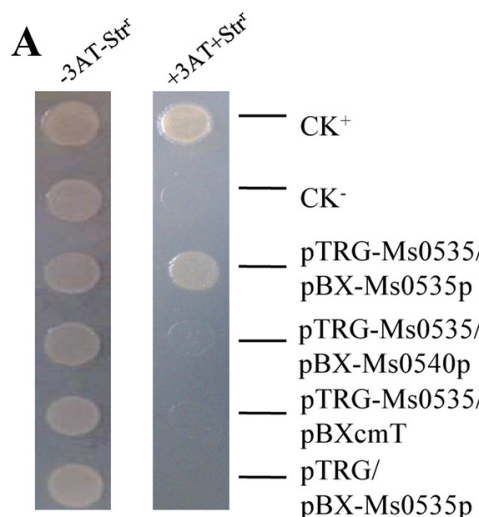

C

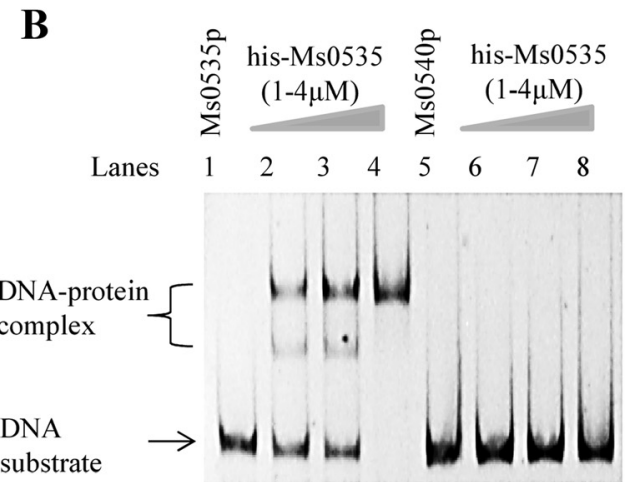

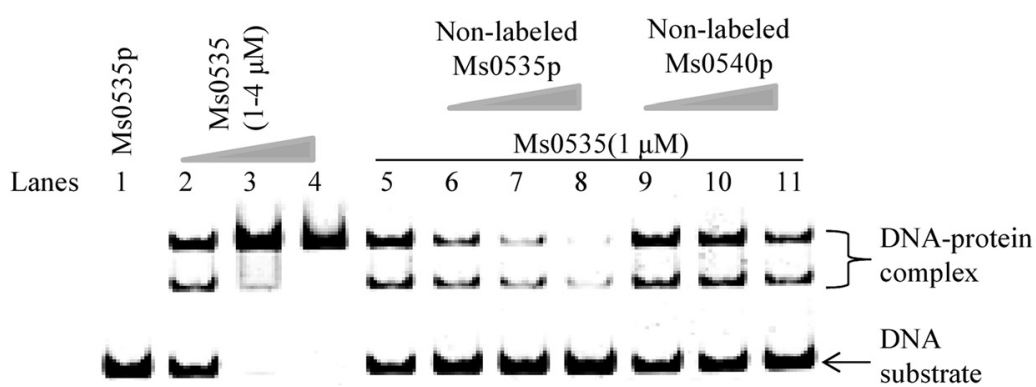

Fig. 2 Ms0535 specifically binds to its own promoter. (a) Bacterial one-hybrid assays. Promoters of the Ms0535 and Ms0540 genes were cloned into the pBXcmT vector, and the Ms0535 gene was cloned into the pTRG vector. A pair of pBXcmT/pTRG plasmids was co-transformed into the reporter strain, and then its growth was tested together with the self-activation controls on a selective medium. Co-transformants containing the pBX-Rv2031/pTRG-Rv3133 plasmids [26] served as positive controls (CK+), and co-transformants containing the empty vectors pBXcmT and pTRG served as negative controls (CK-). (b) Electrophoretic mobility shift assays (EMSAs). The Ms0535p (lanes 1-4) and Ms0540p (lanes 5-8) DNA substrates were co-incubated with various amounts of the Ms0535 protein. The free DNA substrate and DNA-protein complexes are indicated. (c) EMSAs for the specific binding of Ms0535 to its own promoter. Then, $0.05 \mathrm{nM}$ of fluorescein isothiocyanate-labeled Ms0535 promoter DNA substrate was co-incubated with the Ms0535 protein in the absence (lanes 1-5) or presence of non-labeled Ms0535p (0.25-1 nM) (lanes 6-8) or non-labeled Ms0540p (0.25-1 nM) (lanes 9-11). Unlabeled Ms0535 and unlabeled Ms0540 promoter DNA substrates were used to compete with the labeled Ms0535 promoter DNA. The Ms0535 promoter, but not the Ms0540 promoter, inhibited the binding of Ms0535 to the labeled Ms0535 promoter DNA substrate

performed. Unlabeled Ms0535p and Ms0540p promoters were used to compete with labeled $M s 0535 p$, and $M s 0535 p$, but not $M s 0540 p$, could competitively inhibit the binding of Ms0535 to the labeled Ms0535p promoter (Fig. 2c).

These findings strongly suggest that Ms0535 can specifically bind to its own promoter, $M s 0535 p$.

\section{Ms0535 recognizes a palindromic sequence motif}

To identify the DNA-binding site for Ms0535, a DNase I footprinting assay was performed as described previously [28]. The results showed that Ms0535 protected the sequence 5'-CCTTGCATGCATTCTTGCATGCAAGG-3' in the $M s 0535 p$ promoter (Fig. 3a). The protected DNA region extended from positions -19 to -44 (assuming the Ms0535 start codon to be +1 ) in the coding strand (Fig. 3b). In this region, a palindromic motif formed by two IRs (5'-CCTTGCATGCA-3'), which are separated by $4 \mathrm{nt}$, was found (Fig. 3b). To detect binding between Ms0535 and the binding motif, short oligonucleotides were synthesized, and EMSAs were conducted. In the experiments, the wild-type motif and three mutants were included (Fig. 3c). The results showed that Ms0535 bound the wild-type $M s 0535 p 0$ substrate, resulting in shifted bands (Fig. 3c, lanes 1-4). In addition, Ms0535 also bound to $M s 0535 p 1$ and $M s 0535 p 2$, in which one IR was mutated (Fig. 3c, lanes 5-8 and 9-12, respectively). In contrast, Ms0535 did not bind the Ms0535p7 fragment, in which both IRs were mutated (Fig. 3c, lanes 13-16). Interestingly, when Ms0535 bound to Ms0535p1 and Ms0535p2, the single IR mutants, only one shifted band was observed (Fig. 3c, lanes 5-8 and 9-12, respectively), suggesting that Ms0535 may bind the promoter in two steps.

In conclusion, these results indicate that Ms0535 recognizes a 26-bp palindromic sequence motif.

The Ms0535 and Ms0534 genes reside in an Ms0534-0535 operon

Because Ms0535 binds to a 26-bp sequence motif in the upstream region of its own promoter, we created a system 


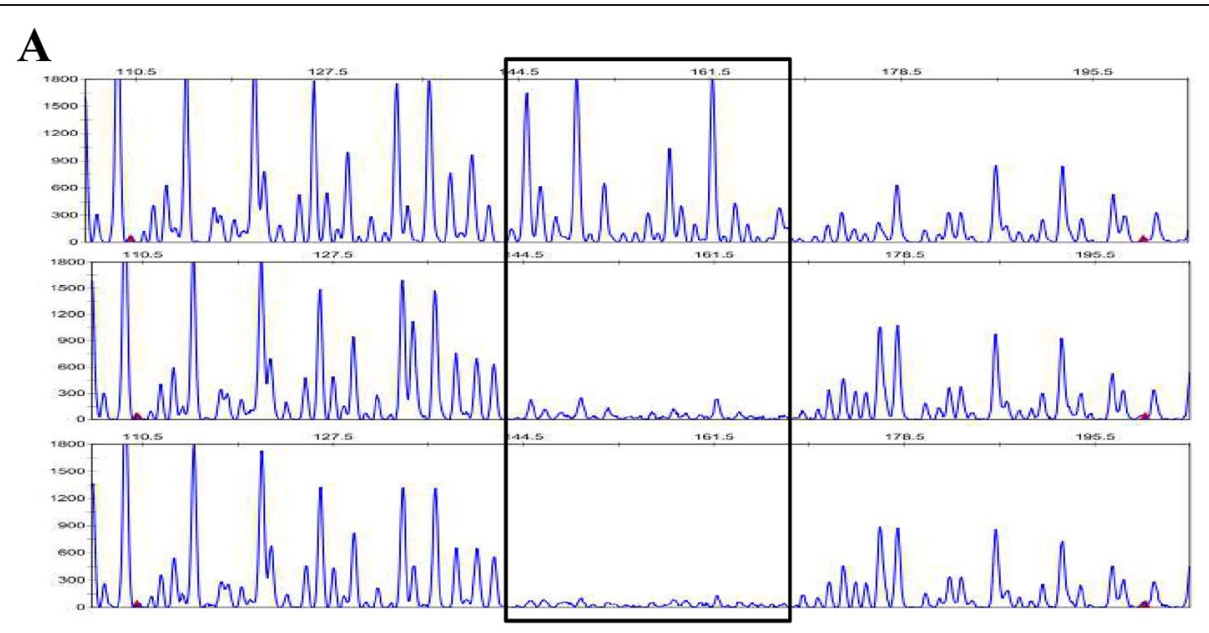

B

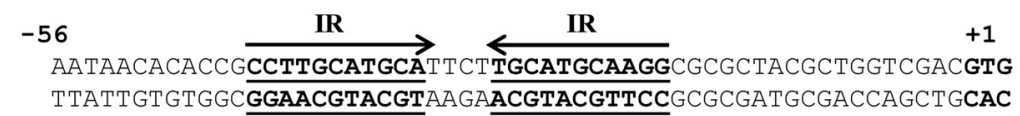

Ms0535 start codon

C Ms0535P0:

5' - CCGCCTTGCATGCATT

CTTGCATGCAAGGCGC-3'

Ms0535P1:

5'-CCGTTCCATGCATGTT

CTTGCATGCAAGGCGC-3'

Ms0535P2:

5'-CCGCCTTGCATGCATT

CTCATGCATGGCCCGC-3'

Ms0535P7:

5'-CCGTTCCATGCATGTT

CTCATGCATGGCCCGC-3'

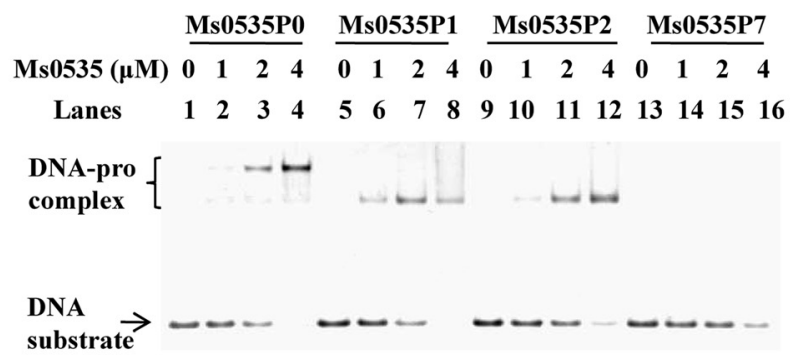

D

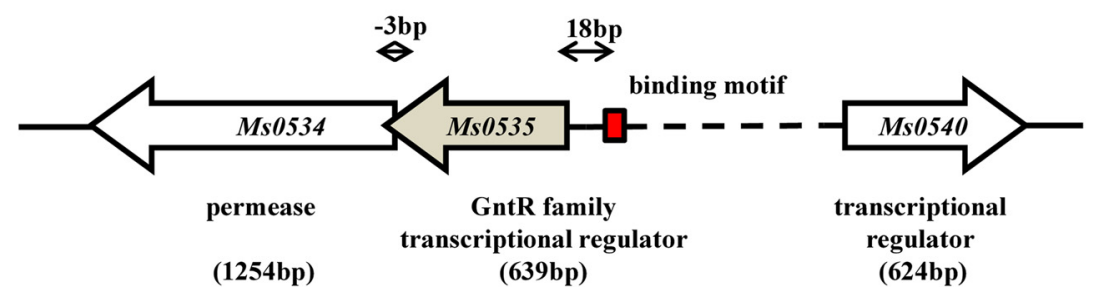

Fig. 3 DNA-binding motif assays for Ms0535. (a) Dye primer sequencing based on a DNase I footprinting assay. The Ms0535 promoter DNA was digested with DNasel in the presence of increasing amounts of Ms0535. The protected regions are indicated by a black frame. (b) Sequence and structural characteristics of the protected Ms0535 promoter region. The regions protected by Ms0535 are underlined. The binding motif is a 26 -bp sequence containing invert repeats (IRs) with a 4-bp spacer. The translational start codon of Ms0535 is indicated in bold. (c) Electrophoretic mobility shift assays of the DNA-binding activity of Ms0535 towards DNA substrates with (lanes 1-4) or without (lanes 13-16) the IR sequences. The DNA substrates were individually incubated with 0-4 $\mathrm{MM}$ of the Ms0535 protein. (d) Analysis of the domain structure of Ms0535 and the genomic location of its DNA binding motif. The Ms0535 gene encodes a typical GntR regulator, and it shares a common upstream promoter region with the major facilitator superfamily permease Ms0534. The distance between the motif and the coding sequence of Ms0535 is 18 bp

to locate the putative promoter and to detect the operator region of Ms0535 (Fig. 3d). A genomic location analysis suggested that Ms0535 shares the same upstream DNA region with the MFS permease Ms0534. Subsequent reverse transcription-PCR (RT-PCR) assays showed that the two genes were co-transcribed (Fig. 4). The cDNA used in this experiment was reverse transcribed by a reverse primer that was complementary to a region within the
Ms0535 gene. DNA fragments encompassing the Ms0534 and MS0535 genes, as well as the region between the genes, can be amplified from the cDNA template. An mRNA template and the amplification of an unrelated DNA fragment encompassing the $\mathrm{Ms} 0540$ gene were used as negative controls. This result suggests that the Ms0534 and Ms0535 genes are co-transcripted and reside in an Ms0534-Ms0535 operon in $M$. smegmatis. 

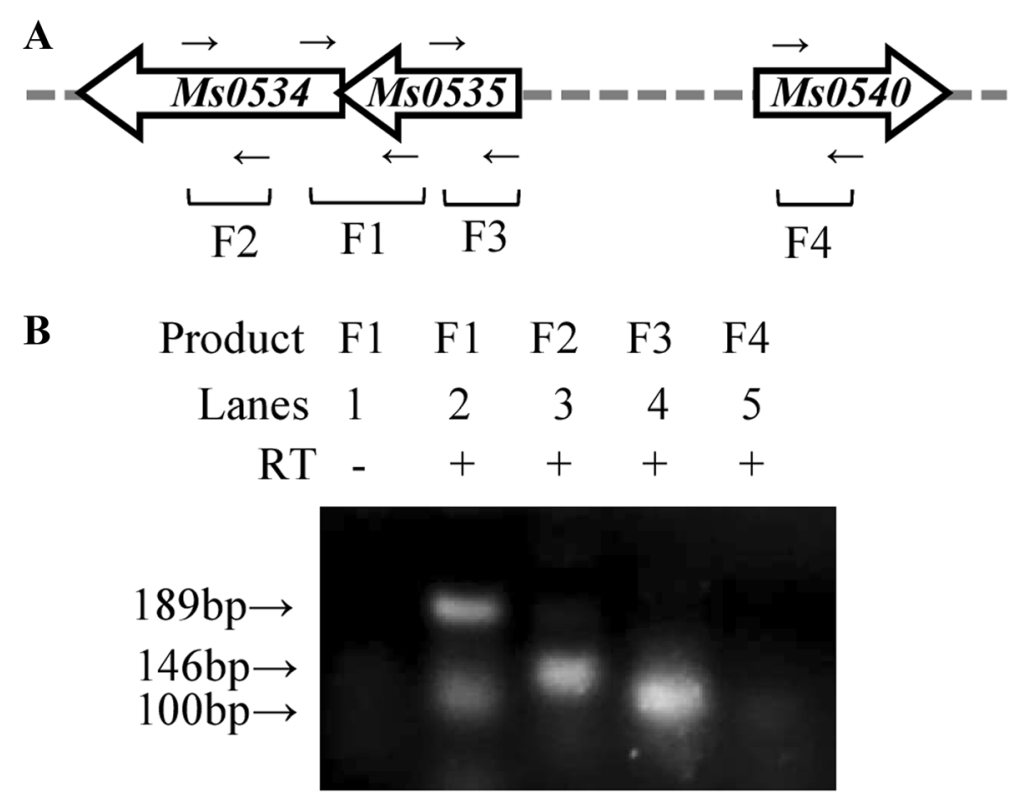

Fig. 4 Assays for Ms0534-Ms0535 co-transcription by reverse transcription-PCR. (a) The schematic operon structure of Ms0534-Ms0535. Primers designed for the assays are indicated by black arrows; F1 primers are complementary to the sequences of the two adjacent genes. (b) Reverse transcription-PCR assays for Ms0534-Ms0535 co-transcription. In this assay, the resulting CDNA was transcribed by the F3 reverse primer. For the F1 fragment, mRNA (DNA-free) was used as a negative control (lane 1). In lane 2, a 189-bp reverse transcription-PCR product indicates the co-transcription of Ms0534-Ms0535. The 146-bp F2 and 100-bp F3 extension products are shown in lanes 2 and 3. The negative result in lane 5 reveals that the F4 fragment cannot be amplified from the CDNA template. The PCR procedure was as follows: the reactions underwent 35 cycles of denaturation at $95^{\circ} \mathrm{C}$ for $30 \mathrm{~s}$, annealing at $60{ }^{\circ} \mathrm{C}$ for $30 \mathrm{~s}$, and extension at $72{ }^{\circ} \mathrm{C}$ for $30 \mathrm{~s}$

\section{Ms0535 positively regulates its own expression and the expression of $\mathrm{Ms} 0534$}

To characterize the biological function of Ms0535, we constructed a $M$. smegmatis Ms0535 knockout strain using a gene replacement strategy (Additional file 1: Figure S1). Subsequently, the expression of the adjacent gene, Ms0534, in both the wild-type and Ms0535 deletion strains was compared by quantitative RT-PCR (qRT-PCR) assays. As shown in Fig. 5a, the expression of $\mathrm{Ms} 0534$ was significantly down-regulated in the Ms0535 knockout strain compared with that in the wild-type strain. In contrast, the expression of the negative control gene Ms0540, whose promoter does not contain a conserved binding motif, did not show any obvious changes. This finding suggested that Ms0535 could function as a positive regulator. Consistent with this view, when $M s 0535$ was overexpressed (by approximately six-fold), the expression of $\mathrm{Ms} 0534$ was significantly up-regulated (Fig. 5b). These results indicate that Ms0535 can function as a positive regulator of Ms0535/ Ms0534 in M. smegmatis.

As we proved, overexpressing Ms0535 can increase the INH resistance of $M$. smegmatis. To determine whether INH can affect the expression of Ms0535, qRT-PCR experiments were performed on the wild-type strain after cultivation in the presence or absence of INH for $6 \mathrm{~h}$ (Fig. 5c). Cultivation in the presence of $10 \mu \mathrm{g} / \mathrm{mL}$ INH did not alter the expression of the Ms0534 and Ms0535 genes.
However, in the presence of $30 \mu \mathrm{g} / \mathrm{mL}$ INH, the expression of $\mathrm{Ms} 0535$ increased by 1.4-fold relative to bacteria grown in the absence of INH, while $\mathrm{Ms} 0534$ expression increased 1.6-fold. Since these differences in expression were not very significant, we concluded that these two genes are not INH-responsive genes, although overexpression of either Ms0535 or Ms0534 increases INH resistance (see below).

\section{Ms0535 regulates $M$. smegmatis antibiotic resistance via the putative permease Ms0534}

Ms0534 has been predicted to encode a permease that is possibly involved in drug export. Moreover, using the sequence of the Ms0535 DNA binding motif as a BLAST query, no more binding site was found in the $M$. smegmatis genome, except for the $M s 0535 p$ promoter. Taken together, we hypothesized that Ms0534 should be the effector protein that confers INH resistance in $\mathrm{Ms} 0535$ overexpressing strains. To test this hypothesis, Ms0535 (Additional file 1: Figure S1) and Ms0534 knockout strains (Additional file 2: Figure S2), together with multiple complementary strains, were constructed.

We first determined the growth curves of the wild-type and $\mathrm{Ms} 0534 / \mathrm{Ms} 0535$-overexpressing strains. As shown in Fig. 6a, the Ms0535-overexpressing strain grew much better than the wild-type strain in 7H9 medium containing $10 \mu \mathrm{g} / \mathrm{ml}$ INH (Fig. 6a, left panel), but their growth was 


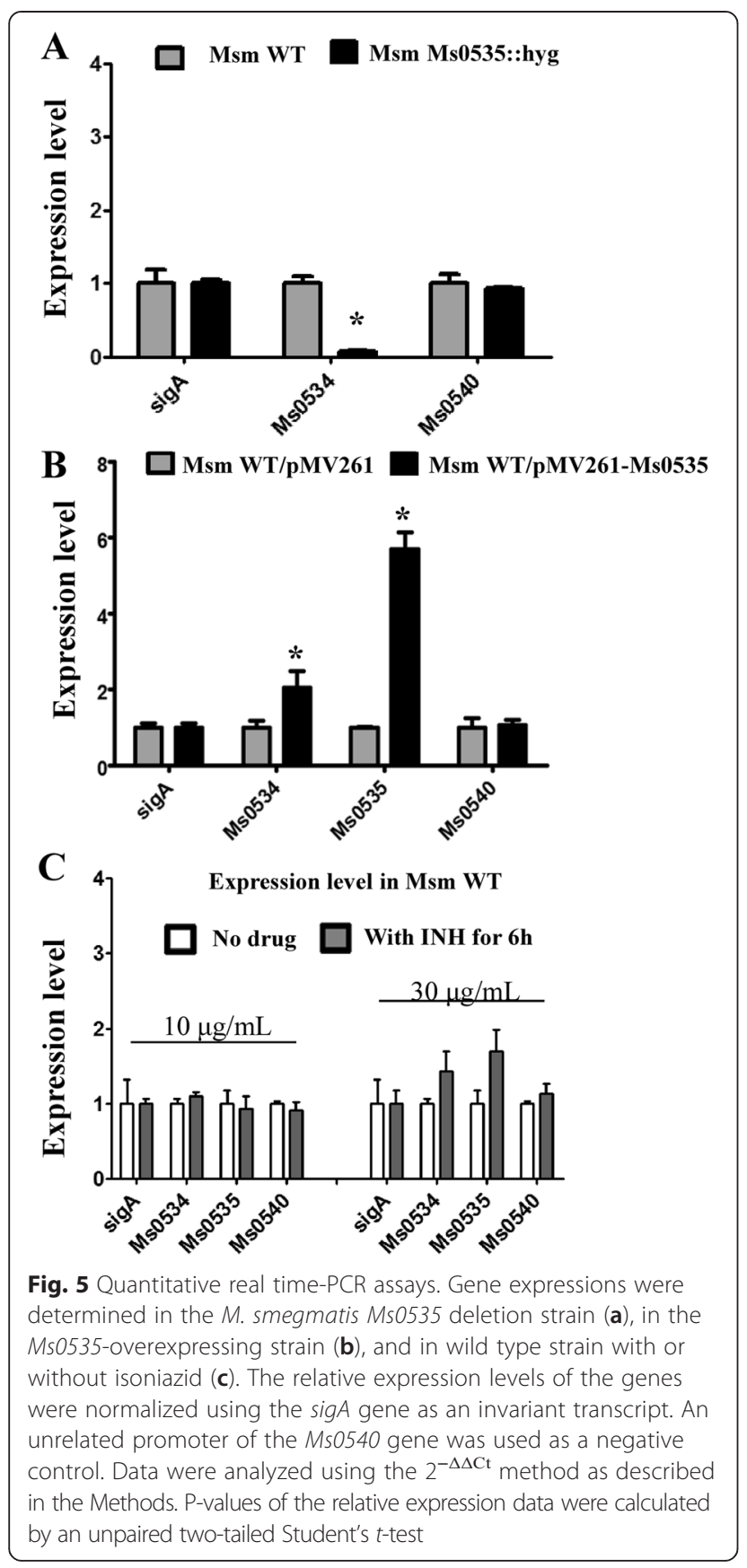

similar in the absence of INH (Additional file 3: Figure S3A, left panel). Comparably, the Ms0534-overexpressing strain grew much better than the wild-type strain in the presence (Fig. 6a, right panel), but not in the absence of INH (Additional file 3: Figure S3A, right panel).

Furthermore, the growth curves of the wild-type, $\mathrm{Ms} 0535$ deletion, and complemented strains were determined. As shown in Fig. 6b, both the Ms0535 (left panel) and Ms0534 deletion strains (right panel) were more sensitive to INH, and their sensitivity could be complemented by inducing the expression of Ms0535 or Ms0534, respectively, using the pMindD system [17]. All these strains grew similarly in the absence of INH (Additional file 3: Figure S3B).

In addition, the $\mathrm{Ms} 0535$ deletion strain was complemented with the $M s 0534$ gene, and the $M s 0534$ deletion strain was complemented with the Ms0535 gene, and the growth curves of these strains were determined with (Fig. 6c) or without (Additional file 3: Figure S3C) INH. As shown in Fig. 6c, complementation with the Ms0534 gene improved the growth of the $\mathrm{Ms} 0535$ deletion strain (Fig. 6c, left). However, complementation with the $\mathrm{Ms} 0535$ gene did not improve the growth of the Ms0534 deletion strain (Fig. 6c, right).

These results support our hypothesis, and they indicate that Ms0535 regulates INH resistance through Ms0534, and Ms0534 is the effector protein that confers INH resistance to the Ms0535-overexpressing strain.

\section{Discussion}

In recent decades, bacteria, especially some important pathogens such as M. tuberculosis, exhibiting drug and multidrug resistance (MDR) have become a major health issue worldwide [29]. In the current study, we showed that a GntR family transcription factor, encoded by the Ms0535 gene, activates the expression of the efflux pump protein Ms0534, an MFS permease, thereby affecting the drug resistance of $M$. smegmatis.

Drug efflux pump proteins play an important role in bacterial drug resistance [30, 31]. Several transcription factors involved in the regulation of these proteins have been characterized [6-8]. Ms2173, a global $\mathrm{Cu}^{2+}$-responsive GntR family transcription factor, was found to be involved in regulating the expression of 37 membrane transport genes, and it was shown to negatively affect mycobacterial drug resistance [18]. GntR family transcription factors, which can regulate MDR, antibiotic biosynthesis, cell permeability, and virulence [5, 32], are widely distributed in bacteria [26]; GntR is named after a repressor of the B. subtilis gluconate operon [5]. The family members usually function as transcriptional repressors, although there are also reports of GntR activators, such as S. marcescens PigT [18], E. faecalis CitO [19], and B. subtilis GabR [25]. The genomes of mycobacteria contain a large number of GntR family transcription factors [11], although little is known about the function of the other members of this family. In this study, we isolated and characterized the GntR/FadR subfamily transcriptional factor Ms0535, which acts as an activator and positively affects drug resistance in M. smegmatis. DNaseI footprinting assays and EMSAs confirmed that Ms0535 specifically recognized a 26-bp palindromic sequence motif, which was separated by 4 nt (Fig. 3a).

The MFS is one of the major types of bacterial drug efflux pumps, and it plays an important role in bacterial 

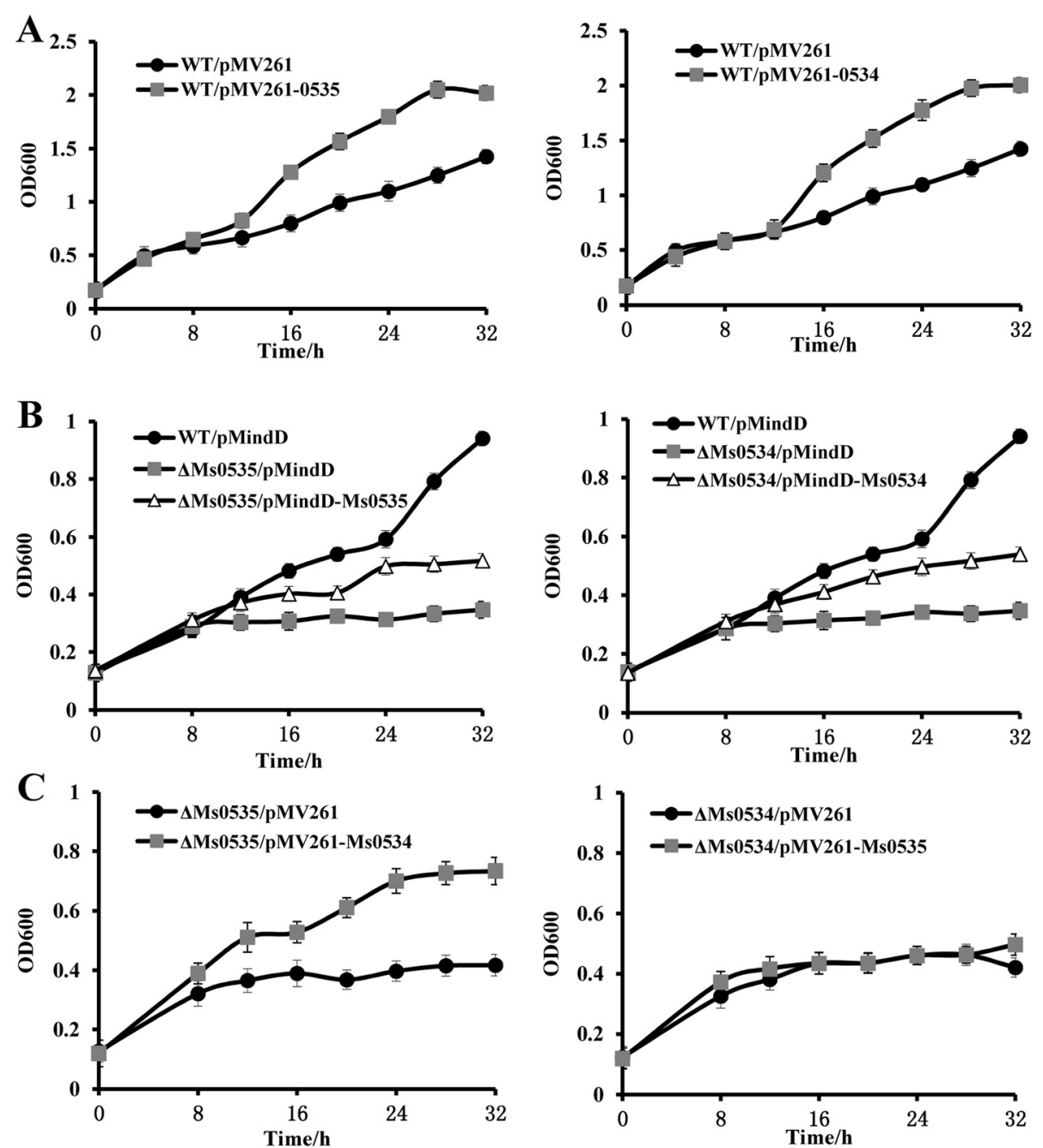

Fig. 6 Determinations of growth curves of M. smegmatis strains. Mycobacterial strains were grown in $7 \mathrm{H} 9$ medium in the presence of $10 \mu \mathrm{g} / \mathrm{ml}$ isoniazid, and growth curves were determined. (a) Left: the wild-type (WT/pMV261) and Ms0535-overexpressing (WT/pMV261-Ms0535) strains; Right: the WT (WT/pMV261) and Ms0534-overexpressing (WT/pMV261-Ms0534) strains. To avoid potential side effects, the empty pMV261 vector was included in the WT strain. (b) Left: the WT (WT/pMindD), Ms0535 deletion ( $\Delta$ Ms0535/pMindD), and $\Delta$ Ms0535 complemented ( $\Delta M$ M 0535/pMindD-Ms0535) strains; Right: the WT (WT/pMindD), Ms0534 deletion ( $\Delta$ Ms0534/pMindD), and $\Delta$ Ms0534 complemented ( $\Delta$ Ms0534/pMindD-Ms0534) strains. To avoid potential side effects, the empty pMindD vector was included in the WT and Ms0534/Ms0535 deletion strains. (c) Left: the Ms0535 deletion ( $\Delta$ Ms0535/pMV261) strain and the $\Delta$ Ms0535 strain complemented with Ms0534 (UMs0535/pMV261-Ms0534); Right: the Ms0534 deletion strain ( $\Delta$ Ms0534/pMV261) and the $\Delta$ Ms0534 strain complemented with Ms0535 (AMs0534/pMV261-Ms0535). To avoid potential side effects, the empty pMV261 vector was included in the Ms0534/Ms0535 deleted strains. Error bars represent the standard deviation of three biological replicates

drug efflux $[4,5]$. The current study showed that most of the mycobacterial drug efflux pumps belong to the MFS. This confirmed that the transporters of the MFS play an important role in mycobacterial drug resistance [33]. LfrA is a transporter protein that belongs to the MFS, and it is mainly involved in the efflux of EB and acriflavine in bacteria. It is the first drug efflux pump been discovered in M. tuberculosis [8]. Ms0534 is a permease that belongs to the MFS. It is located in the same operon as the GntR family transcriptional factor Ms0535. In this study, we found that Ms0535 activates the expression of Ms0534 (Fig. 5a). An interesting finding from the present work was the determination that both Ms0535and Ms0534-overexpressing mycobacterial strains exhibited higher levels of INH resistance than the wild-type strain (Fig. 6a left and right panels, respectively). Conversely, the $\mathrm{Ms} 0535$ and $\mathrm{Ms} 0534$ deletion strains were more sensitive to INH than the wild-type strain (Fig. 6a right panel; Fig. 6b, left panel). Furthermore, we found that overexpressing $\mathrm{Ms} 0534$ in the $\mathrm{Ms} 0535$ deletion strain enhanced mycobacterial INH resistance (Fig. 6b, middle panel). However, overexpressing Ms0535 in the Ms0534 
deletion strain did not affect INH resistance (Fig. 6b, right panel). This suggests that Ms0535 acts as a transcriptional activator that regulates the expression of the drug efflux pump protein Ms0534, which positively regulates the drug resistance of $M$. smegmatis.

It is noteworthy that although the complementation strains grew better than the deletion strains under INH stress, their resistance to INH are still far from the wildtype control (Fig. 6b). One probable reason for this result is that the corresponding promoter activities are different in the pMindD vector and the M. smegmatis genome. Another possibility is that induce of hyg gene may cause polar effect in gene expression. Using a scarless gene deletion may avoid this effect by removing antibiotic resistance genes with phage infection [34].

As is well known, INH is active exclusively against mycobacteria, but pathogenic $M$. tuberculosis and nonpathogenic $M$. smegmatis display greatly different sensitivities to INH. Compared with its activity against $M$. tuberculosis, INH is 100 times less active against $M$. smegmatis [35]. Additionally, genomic variations between the two species may also be responsible for this difference. The M. smegmatis genome encodes more than 500 potential regulatory factors; however, only 214 regulatory factors are encoded by the M. tuberculosis genome. Ms0535 is a GntR family transcription factor encoded by the M. smegmatis genome, while absent in $M$. tuberculosis. Furthermore, its target Ms0534, a putative permease, is absent in M. tuberculosis as well. According to our results, these two genes may contribute the drug resistance of $M$. smegmatis and this partially explain the difference in INH resistance between the two mycobacterial species.

Studies have shown that MFS proteins can transport molecules such as drugs, glucide, and phosphate [5]. Consistently, we showed that overexpression of the transcription factor Ms0535 and the MFS permease Ms0534 affects the INH resistance of $M$. smegmatis. Additionally, no potential Ms0535 target genes were identified by searching the M. smegmatis with the sequence of the Ms0535 DNA binding motif. This suggests that Ms0534 may transport $\mathrm{INH}$, and that Ms0535 specifically regulates this process.

\section{Conclusions}

In the present study, a GntR family, VanR-like transcriptional factor, Ms0535, was shown to be a transcription activator and to positively regulate INH resistance of $M$. smegmatis. Ms0535 specifically recognized a 26-bp palindromic sequence motif separated by a 4-nt spacer, and it activated its own expression, as well as that of the Ms0534 efflux pump gene in the same operon. Our findings establish Ms0535 as a novel GntR family activator in mycobacteria, and they significantly enhance our understanding of the regulatory mechanism of bacterial drug resistance.

\section{Methods}

\section{Strains, enzymes, plasmids and reagents}

E. coli BL21 cells and pET28a vector were purchased from Novagen (Darmstadt, Germany). pBT, pTRG vectors and $E$. coli XR host strains were purchased from Stratagene (La Jolla, CA, USA) (Additional file 4: Table S1). Restriction enzymes, T4 ligase, Modification enzymes, Pyrobest DNA polymerase, dNTPs and all antibiotics were obtained from TaKaRa Biotech (Shiga, Japan). The reagents for one-hybrid assay were purchased from Stratagene. Polymerase Chain Reaction (PCR) primers were synthesized by Invitrogen (Carlsbad, CA, USA) (Additional file 4: Table S2).

\section{The screening of isoniazid related transcriptional regulators}

Over 500 transcriptional regulator genes were amplified from $M$. smegmatis genomic DNA. The gene fragments were mixed as a pool and cloned into pMV261 vector [36] to construct the regulatory genes overexpression plasmids library. The plasmids library were electrophoretic transferred into M. smegmatis mc2 155, and the strains were screened on $7 \mathrm{H} 10$ plates containing $20 \mu \mathrm{g} / \mathrm{mL}$ INH. As a result, those having increased INH resistance or decreased INH susceptibilities were identified as primary candidates. To avoid random mutations that may contribute to INH resistance, plasmid were extracted from each of the primary candidates, and transformed into the wild type $M$. smegmatis and assayed thrice in a similar way. In final, the increased INH resistance is sufficient to attribute to the overexpression of the corresponding transcriptional regulator.

\section{Bacterial one-hybrid assay}

Bacterial One-Hybrid assays were carried out as described previously [27]. Ms0535 was cloned into the pTRG vector (Stratagene). Promoters (500 bp upsteam of the open reading frame) of the M. smegmatis genes were amplified using appropriate primers (Additional file 4: Table S2) and cloned into the $\mathrm{pBXcmT}$ vector. The selective plate contains $20 \mathrm{mM}$ 3-AT (3-amino-1,2,4-triazole), $16 \mu \mathrm{g} / \mathrm{ml}$ streptomycin, $15 \mu \mathrm{g} / \mathrm{ml}$ tetracycline, $34 \mu \mathrm{g} / \mathrm{ml}$ chloramphenicol and $30 \mu \mathrm{g} / \mathrm{ml}$ kanamycin. A co-transformant containing pBX-Rv2031/pTRG-Rv3133 plasmids was served as positive control $(\mathrm{CK}+)$ and a co-transformant containing empty vector pBX and pTRG was served as negative control (CK-). A co-transformant containing pTRG-Ms0535/ pBXcmT plasmids and pTRG/pBX-Ms0535p plasmids were served as self-activated controls. The plates were incubated at $30{ }^{\circ} \mathrm{C}$ for $3-4$ days.

\section{Cloning, expression and protein purification}

Cloning, expression and protein purification were performed as previously described [37]. M. smegmatis mc2 155 genes were amplified using specific primers 
(Additional file 4: Table S2). Ms0535 was cloned into the pET28a vector to produce recombinant vectors (Additional file 4: Table S1).

\section{Electrophoretic mobility shift assay (EMSA)}

DNA fragments for the DNA-binding activity assays were amplified by PCR from $M$. smegmatis genomic DNA using their primers labeled with Fluorescein Isothiocyanateor (FITC) or directly synthesized (Additional file 4: Table S2). The EMSA assays were conducted as previously described [37].

\section{DNase I footprinting assay}

The $300 \mathrm{bp}$ promoter region of the Ms0535 gene (Additional file 4: Table S1) was amplified by PCR using appropriate primers labeled with Fluorescein Isothiocyanate (FITC) (Additional file 4: Table S2). The amplified products were purified with BioFlux PCR DNA Purification kit (BioFlux) and then subjected to similar binding reaction as in EMSA. DNaseI footprinting was performed using the method of dye primer sequencing $[38,39]$.

\section{Recombinant strains}

Knockout of the Ms0535 and Ms0534 gene in M. smegmatis was performed as described previously [38, 40]. Deletion of Ms0535 and Ms0534 were further verified by Southern blotting [39]. Primers used in the assays were provided in Additional file 4: Table S1.

Ms0534 and Ms0535 genes were amplified from $M$. smegmatis genomic DNA by their respective primers (Additional file 4: Table S2). To overproduce in M. smegmatis, Ms0534 gene was inserted downstream of the hsp60 promotor of pMV261 [36]. And the Ms0534 and Ms0535 genes were cloned into a pMind vector [41] for complementing in knockout strains. The recombinant plasmids were electroporated into M. smegmatis mc2 155 and selected on 7H10 medium containing $30 \mathrm{mg} / \mathrm{mL}$ kanamycin.

\section{Quantitative real time PCR (qRT-PCR)}

qRT-PCR were performed as described previously [38]. Isolation of mRNA and cDNA preparation of wild type strains, deletion mutants and overexpression M. smegmatis strains were performed and real-time PCR analysis was subsequently carried out according to previously described procedures [37]. Each PCR reaction $(20 \mu \mathrm{l})$ contained $2 \mu \mathrm{l}$ of $2 \times$ SYBR Green Master Mix Reagent (Applied Biosystems), $1.0 \mu \mathrm{l}$ of cDNA samples and $200 \mathrm{nM}$ gene-specific primers (Additional file 4: Table S3). Gene expression levels were normalized to the levels of sigA rRNA gene transcripts and relative expression changes were calculated using the $2-\Delta \Delta \mathrm{Ct}$ method [38, 42].

\section{Determination of mycobacterial growth curves}

Growth patterns of the wild-type (Msm) mycobacterial strain, Ms0535-deleted mutant (Msm/Ms0535::hyg), Ms0534-deleted mutant (Msm/Ms0534::hyg), the overexpression (Msm/pMV261-Ms0535, Msm/pMV261-Ms0534) mycobacterial strains and complemented strains $(\mathrm{Msm} /$ pMindD-Ms0535, Msm/pMindD-Ms0534) were determined according to previously described [9].

\section{Availability of supporting data}

The data sets supporting the results of this article are included within the article and its additional files.

\section{Additional files}

\begin{abstract}
Additional file 1: Figure S1. Construction of the M. smegmatis Ms0535 knockout strain and Southern blotting assay. (A) Schematic of the recombination strategy for the deletion of the Ms0535 gene from the M. smegmatis genome. (B) A map of the recombinant vector pMind-Ms0535 containing the upstream and downstream sequences of Ms0535 and the gene that confers resistance against hygromycin. (C) Schematic of the DNA fragments of the wild-type and $\Delta$ Ms0535 knockout strains treated with the restriction enzyme Smal. The probe is indicated with a black bar. (D) Southern blot assays. A 300-bp probe corresponding to the sequences of the Ms0535 upstream genomic fragment of $M$. smegmatis was obtained by PCR and labeled with digoxigenin dUTP (Roche, Mannheim Germany). The probe was used to detect changes in the size of the Smal-digested genomic fragment of $M$. smegmatis before and after recombination. (TIFF $942 \mathrm{~kb}$ )
\end{abstract}

Additional file 2: Figure S2. Construction of the M. smegmatis Ms0534 knockout strain and Southern blotting assays. (A) Schematic of the recombination strategy for the deletion of the $\mathrm{Ms0534}$ gene from the $\mathrm{M}$. smegmatis genome. (B) A map of the recombinant vector pMind-Ms0534 containing upstream and downstream sequences of Ms0534 and the gene that confers resistance against hygromycin. (C) Schematic representation of the DNA fragments of the wild-type and $\Delta$ Ms0534 knockout strains treated with the restriction enzyme Narl. The probe is indicated with a black bar. (D) Southern blot assays. A 300-bp probe corresponding to the sequences of the Ms0534 upstream genomic fragment of $M$. smegmatis was obtained by PCR and labeled with digoxigenin dUTP (Roche). The probe was used to detect changes in the size of the Narl-digested genomic fragment of M. smegmatis before and after recombination. (TIFF $871 \mathrm{~kb}$ )

Additional file 3: Figure S3. Growth curves of M. smegmatis strains in the absence of isoniazid. The strains are the same as those described in Fig. 6. (TIFF $228 \mathrm{~kb}$ )

Additional file 4: Table S1. Strains and plasmids used in this study. Table S2. Primers used in this study. Table S3. Primers used for quantitative real time-PCR and reverse transcription PCR. (DOC $67 \mathrm{~kb}$ )

\section{Abbreviations}

3-AT: 3-amino-1,2,4-triazole; qRT-PCR: Quantitive real-time PCR; ABC: ATPbinding cassette superfamily; EB: Ethidium bromide; EMB: Ethambutol; EMSA: electrophoretic mobility shift assay; FITC: Fluorescein Isothiocyanateor; INH: Isoniazid; MFS: Major facilitator superfamily; MATE: Multidrug and toxic compound extrusion family; MDR: Multi-drug resistance; RIF: Rifampicin; RND: Resistance/nodulation/cell division family; SMR: Small multidrug resistance family.

\section{Competing interests}

The authors declare that they have no competing interests.

\section{Authors' contributions}

MY conceived the study, coordinated the experimental work and, along with $\mathrm{LZ}$ and $\mathrm{J}$, wrote the manuscript. Both $\mathrm{JH}$ and $\mathrm{LZ}$ carried out the experimental work. All authors read and approved the final manuscript. 


\section{Acknowledgements}

This work was supported by the National Natural Science Foundation of China (31301079), Financial Grant from the China Postdoctoral Science Foundation (2013 M540587 and 2014 T70709).

\section{Author details}

${ }^{1}$ National Key Laboratory of Agricultural Microbiology, Center for Proteomic Research, College of Life Science and Technology, Huazhong Agricultural University, Wuhan 430070, China. ${ }^{2}$ College of Life Science and Technology, Huazhong Agricultural University, Wuhan 430070, China.

\section{Received: 23 March 2015 Accepted: 8 October 2015}

\section{Published online: 16 October 2015}

\section{References}

1. Li X-Z, Nikaido H. Efflux-mediated drug resistance in bacteria: an update. Drugs. 2009;69:1555-623.

2. Alekshun MN, Levy SB. Molecular mechanisms of antibacterial multidrug resistance. Cell. 2007;128:1037-50.

3. Walsh C. Molecular mechanisms that confer antibacterial drug resistance. Nature. 2000;406:775-81.

4. Yan N. Structural advances for the major facilitator superfamily (MFS) transporters. Trends Biochem Sci. 2013;38:151-9.

5. Pao SS, Paulsen IT, Saier MH. Major facilitator superfamily. Microbiol Mol Biol Rev MMBR. 1998;62:1-34.

6. Grkovic S, Brown MH, Roberts NJ, Paulsen IT, Skurray RA. QacR is a repressor protein that regulates expression of the Staphylococcus aureus multidrug efflux pump QacA. J Biol Chem. 1998;273:18665-73.

7. Ahmed M, Borsch CM, Taylor SS, Vázquez-Laslop N, Neyfakh AA. A protein that activates expression of a multidrug efflux transporter upon binding the transporter substrates. J Biol Chem. 1994:269:28506-13.

8. Buroni S, Manina G, Guglierame P, Pasca MR, Riccardi G, De Rossi E. LfrR is a repressor that regulates expression of the efflux pump LfrA in Mycobacterium smegmatis. Antimicrob Agents Chemother. 2006;50:4044-52.

9. Yang $M$, Gao C, Wang $Y$, Zhang H, He Z-G. Characterization of the interaction and cross-regulation of three Mycobacterium tuberculosis RelBE modules. PloS One. 2010;5, e10672.

10. Li Y, Zeng J, Zhang H, He Z-G. The characterization of conserved binding motifs and potential target genes for $M$. tuberculosis MtrAB reveals a link between the two-component system and the drug resistance of $\mathrm{M}$. smegmatis. BMC Microbiol. 2010;10:242.

11. Cole ST, Brosch R, Parkhill J, Garnier T, Churcher C, Harris D, et al. Deciphering the biology of Mycobacterium tuberculosis from the complete genome sequence. Nature. 1998;393:537-44.

12. Li X-Z, Zhang L, Nikaido H. Efflux pump-mediated intrinsic drug resistance in Mycobacterium smegmatis. Antimicrob Agents Chemother. 2004;48:2415-23.

13. De Rossi E, Aínsa JA, Riccardi G. Role of mycobacterial efflux transporters in drug resistance: an unresolved question. FEMS Microbiol Rev. 2006;30:36-52.

14. Louw GE, Warren RM, van Pittius NC G, McEvoy CRE, Van Helden PD, Victor TC. A balancing act: efflux/influx in mycobacterial drug resistance. Antimicrob Agents Chemother. 2009;53:3181-9.

15. Haydon DJ, Guest JR. A new family of bacterial regulatory proteins. FEMS Microbiol Lett. 1991;63:291-5.

16. Vindal V, Ranjan S, Ranjan A. In silico analysis and characterization of GntR family of regulators from Mycobacterium tuberculosis. Tuberc Edinb Scotl. 2007:87:242-7.

17. Rao M, Liu H, Yang M, Zhao C, He Z-G. A copper-responsive global repressor regulates expression of diverse membrane-associated transporters and bacterial drug resistance in mycobacteria. J Biol Chem. 2012;287:39721-31.

18. Fineran PC, Everson $L$, Slater $H$, Salmond GPC. A GntR family transcriptional regulator (PigT) controls gluconate-mediated repression and defines a new, independent pathway for regulation of the tripyrrole antibiotic, prodigiosin, in Serratia. Microbiol Read Engl. 2005;151(Pt 12):3833-45.

19. Blancato VS, Repizo GD, Suárez CA, Magni C. Transcriptional regulation of the citrate gene cluster of Enterococcus faecalis Involves the GntR family transcriptional activator CitO. J Bacteriol. 2008;190:7419-30.

20. DiRusso CC, Nyström T. The fats of Escherichia coli during infancy and old age: regulation by global regulators, alarmones and lipid intermediates. Mol Microbiol. 1998;27:1-8.

21. Lord DM, Uzgoren Baran A, Soo WW, Wood TK, Peti W, Page R. McbR/YncC: implications for the mechanism of ligand and DNA binding by a bacterial
GntR transcriptional regulator involved in biofilm formation. Biochemistry. 2014:53:7223-31.

22. Truong-Bolduc QC, Hooper DC. The transcriptional regulators NorG and Mgra modulate resistance to both quinolones and beta-lactams in Staphylococcus aureus. J Bacteriol. 2007;189:2996-3005

23. Hoskisson PA, Rigali S. Chapter 1: Variation in form and function the helixturn-helix regulators of the GntR superfamily. Adv Appl Microbiol. 2009;69:1-22.

24. Guzmán K, Badia J, Giménez R, Aguilar J, Baldoma L. Transcriptional regulation of the gene cluster encoding allantoinase and guanine deaminase in Klebsiella pneumonia. J Bacteriol. 2011;193:2197-207.

25. Edayathumangalam R, Wu R, Garcia R, Wang Y, Wang W, Kreinbring CA, et al. Crystal structure of Bacillus subtilis GabR, an autorepressor and transcriptional activator of gabT. Proc Natl Acad Sci U S A. 2013;110:17820-5.

26. Rigali S, Derouaux A, Giannotta F, Dusart J. Subdivision of the helix-turn-helix GntR family of bacterial regulators in the FadR, HutC, MocR, and YtrA subfamilies. J Biol Chem. 2002:277:12507-15.

27. Guo M, Feng H, Zhang J, Wang W, Wang Y, Li Y, et al. Dissecting transcription regulatory pathways through a new bacterial one-hybrid reporter system. Genome Res. 2009;19:1301-8.

28. Zianni M, Tessanne K, Merighi M, Laguna R, Tabita FR. Identification of the DNA bases of a DNase I footprint by the use of dye primer sequencing on an automated capillary DNA analysis instrument. J Biomol Tech JBT. 2006:17:103-13.

29. World Health Organization. Global Tuberculosis Control WHO Report 2010 Geneva: World Health Organization; 2010.

30. Nikaido $H$, Zgurskaya $H I$. AcrAB and related multidrug efflux pumps of Escherichia coli. J Mol Microbiol Biotechnol. 2001;3:215-8.

31. Ryan BM, Dougherty TJ, Beaulieu D, Chuang J, Dougherty BA, Barrett JF. Efflux in bacteria: what do we really know about it? Expert Opin Investig Drugs. 2001;10:1409-22.

32. Subudhi S, Kurdrid P, Hongsthong A, Sirijuntarut M, Cheevadhanarak S, Tanticharoen M. Isolation and functional characterization of Spirulina D6D gene promoter: role of a putative GntR transcription factor in transcriptional regulation of D6D gene expression. Biochem Biophys Res Commun. 2008;365:643-9.

33. De Rossi E, Arrigo P, Bellinzoni M, Silva PAE, Martín C, Aínsa JA, et al. The multidrug transporters belonging to major facilitator superfamily in Mycobacterium tuberculosis. Mol Med. 2002;8:714-24.

34. Xu X, Vilchèze C, Av-Gay Y, Gómez-Velasco A, Jacobs Jr WR. Precise null deletion mutations of the mycothiol synthesis genes reveal their role in isoniazid and ethionamide resistance in Mycobacterium smegmatis. Antimicrob Agents Chemother. 2011;55:3133-9.

35. Vilchèze $C$, Jacobs Jr WR. The mechanism of isoniazid killing: clarity through the scope of genetics. Annu Rev Microbiol. 2007;61:35-50.

36. Snapper SB, Melton RE, Mustafa S, Kieser T, Jacobs WR. Isolation and characterization of efficient plasmid transformation mutants of Mycobacterium smegmatis. Mol Microbiol. 1990;4:1911-9.

37. Yang M, Gao C, Cui T, An J, He Z-G. A TetR-like regulator broadly affects the expressions of diverse genes in Mycobacterium smegmatis. Nucleic Acids Res. 2012;40:1009-20.

38. Wang $Y$, Huang $Y$, Xue $C, H e Y, H e Z-G$. ClpR protein-like regulator specifically recognizes RecA protein-independent promoter motif and broadly regulates expression of DNA damage-inducible genes in mycobacteria. J Biol Chem. 2011;286:31159-67.

39. Bolla JR, Do SV, Long F, Dai L, Su C-C, Lei H-T, et al. Structural and functional analysis of the transcriptional regulator Rv3066 of Mycobacterium tuberculosis. Nucleic Acids Res. 2012;40:9340-55.

40. Blokpoel MC, Murphy HN, O'Toole R, Wiles S, Runn ES, Stewart GR, et al. Tetracycline-inducible gene regulation in mycobacteria. Nucleic Acids Res. 2005;3:e22.

41. Stover CK, de la Cruz VF, Fuerst TR, Burlein JE, Benson LA, Bennett LT, et al. New use of BCG for recombinant vaccines. Nature. 1991;351:456-60.

42. Livak KJ, Schmittgen TD. Analysis of relative gene expression data using real-time quantitative PCR and the 2(-Delta Delta C(T)) Method. Methods San Diego Calif. 2001;25:402-8. 